\section{Outbreak investigation of cholera in Shantinagar VDC of Ilam District in Eastern Nepal}

\author{
Rayamajhi RB,1 Pokharel G,'² Sharma G,² \\ Neupane B, ${ }^{3}$ Khanal VK, ${ }^{1}$ Kafle SU, ${ }^{4}$ Paudel \\ IS, ${ }^{1}$ Pokharel PK ${ }^{1}$
}

${ }^{1}$ School of Public Health and Community Medicine, B. P. Koirala Institute of Health Sciences, Dharan, Nepal, ${ }^{2}$ District Health Office, Ilam, ${ }^{3}$ College of Medical Sciences, Bharatpur, Chitwan, ${ }^{4}$ Department of Pathology, B. P. Koirala Institute of Health Sciences, Dharan, Nepal

*Correspondence to: Dr. Rajan Bikram Rayamajhi, School of Public Health and Community Medicine, B. P. Koirala Institute of Health Sciences, Dharan, Sunsari, Nepal. email: rajan.rayamajhi@hotmail.com, Tel. No.: (+977)-9808667925

\begin{abstract}
INTRODUCTION: In 2012 August, suspected cases of Cholera in increased number were reported to the district health office of Ilam by the in charge of Shantinagar health post asking for intervention to stop further occurrences. The aim of the investigation was to identify the possible source of infection, the causative agent and the application of local control measures for prevention of further recurrences.
\end{abstract}

MATERIALS AND METHODS: Focus group discussion and Key informant survey were conducted to assess the sanitary hygiene and practices along with source of drinking water for probable cause of diarrhea. Similarly, face to face interview was done among the sick and healthy local residents to collect the demographic and clinical details. Ten stool and water samples were sent for analysis in Biratnagar.

RESULTS: 150 local residents had suffered from diarrhea with no deaths. People of all the age group were affected with children being more sufferers. Six of the stool analysis and eight of the water samples were positive for Vibrio Cholerae. It was also observed that sanitary hygiene and practices wasn't adequate after nose and bottoms cleaning of the children and before preparing the meal.

CONCLUSIONS: Lack of adequate cleanliness of the common water tank was found to be the culprit behind the incident and Vibrio Cholerae being the causative agent. Local residents and water management committee were demonstrated the technique of chlorination of water and proper hand washing practices to prevent further recurrences.

KEY WORDS: Epidemic, Water chlorination, Rapid response team, Village development committee

Article submitted 10 August. Reviewed 28 August. Author correction 2 September. Final version accepted 10 September 2013. 


\section{INTRODUCTION}

Cholera, an acute diarrheal disease is caused by the infection of Vibrio Cholera $(01,0139)$ in the intestine and is manifested by sudden onset of profuse painless watery diarrhea (rice water stool), often accompanied by fever and vomiting. This condition led to hypotension, shock and death within few hours to days of the first manifestation. ${ }^{1,2}$ It is a water borne disease that is transmitted by contaminated water and food. ${ }^{1}$ During rainy season, outbreaks of diarrhea diseases are found in various parts of the country. Jajarkot (2009) and Nepalgunj (2008) were some of the examples of diagnosed cholera outbreak. ${ }^{3,4}$ These outbreaks made national headlines in the newspaper and were mostly secondary to lack of adequate hygiene practices.

Shantinagar health post is a government health institution located in a hilly region with difficult geographical terrain. Due to sudden rise in patients suffering from diarrhea more than the expected in the health institution for more than a week; the management committee of the health post felt the need of an intervention from district health office. Thus, district health office epidemic focal person was informed and asked for help. As per the information from the Shantinagar health post to Ilam district health office, a multidisciplinary rapid response team was dispatched for outbreak investigation immediately into the scene. We noted that no deaths had occurred but the local health post was over burdened with the diarrhea cases. Thorough investigation was carried out to assess the magnitude of the problem, identify the source of infection, the causative agent and use of local measures for control and prevention of further recurrences.

\section{MATERIALS AND METHODS}

Loose watery stool diarrhea more than three times a day was defined as a suspected case of Cholera in our study. Similar cases having similar episodes of diarrhea but treated and recovered prior to the intervention by rapid response team were not included in the study. Necessary data were collected by the members of rapid response team with the help of the Shantinagar health post staff. A treatment camp was established in near vicinity of the village for those who can come on their own and home visits were made for the treatment of severe cases by health workers. Five focus group discussion and 12 key informant surveys were done during our visit to the site of outbreak. Written understood consent was taken prior to data collection and confidentiality of the information collected during the visit was maintained.The local water tank and its source were inspected. Water samples were collected from households and the common tank constructed locally from where drinking water was consumed. Stool and water samples were collected avoiding contamination. Stool samples were collected prior to commencement of antibiotics and transported in Cary Blair transport media and Selenite F Broth maintain room temperature to the lab at Biratnagar for routine analysis and culture. Similarly, water samples were also collected and taken to the lab for analysis of coliform and fecal coliform using membrane filter method. The stool and water were collected by the microbiological team for sero typing and taken to their lab in Biratnagar for analysis. Stool culture and use of antisera test was done in our study for identification of serotype. These organisms were identified by agglutination in 0 group 1-specific antiserum directed against the lipopolysaccharide component of the cell wall and by demonstration of their enterotoxigenicity (Bekton Dickinson, USA).

\section{RESULT}

Our study found that more than 256 people were affected by the diarrheal outbreak but only 150 cases were included in our study that fulfilled the inclusion criteria. This finding was much higher than the data of the health post of previous year and other parts of the country and no influx of affected or susceptible population was noted and thus classified as an outbreak.

The first index case was identified on $2^{\text {nd }}$ August and secondary cases were identified subsequently. Secondary cases were identified on the second day of the identification of the index case. It was also noted that most of the cases were family members and friends. Clustering of cases was seen on $3^{\text {rd }}, 4^{\text {th }}$ and $5^{\text {th }}$ day of the outbreak and the gradually the number of cases fell down after the intervention was made by the rapid response team. The control over the number of cases was observed after a week of the intervention in the area of consumption of safe water, cleaning and chlorination of water tank, use of soap and water after defecation, cleaning the nose and bottoms of children and before preparing the meal. The epidemic curve showed the sudden rise and clustering of cases in a short span of time (figure 1). Among the cases, most of them had rice watery stool and few of them also manifested vomiting and fever. Sixty seven percent of them cases were treated with oral rehydration solution, 
medication and home baseed remedies whereas the rest were treated in the temporary treatment camp with intravenous fluids, medication and oral rehydration solution as tolerated. It was also noted that the diarrhea affected people of all the age group but especially children and elderly. The median age of the cases was found to be 19.5 years (Table 1 ). Females were affected more than the males and the clusters of cases were found to have gone to the same school as the children of the school consumed the drinking water of the tank.

Our focus group discussion and key informantion survey revealed that the water tank was hardly cleaned after its construction and water was consumed directly without treating it. It was also observed that outdoor defecation was quite common in that area and the level of awareness among the villagers regarding the sanitary hygiene and practices was quite low. During the interaction it was also noted that, the mothers were less concerned and aware about the importance of hygiene before preparing meals and after cleaning the bottoms and nose of children.

\section{Observational Survey}

During the observation made by the rapid response team, it was noted that the water tank had leakage and was dirty and contaminated with cow dungs and the stream which was the source of water was also contaminated with human defecation. The rain drained the stool into stream which was eventually collected in the water tank.

\section{Lab Analysis}

Results of lab investigation of the stool and water sample were found to be consistent. Six of the stool sample contained causative agent of Vibrio Cholerae and eight water samples contained coliform indicating cross contamination. The stool culture was also found to be positive for Vibrio Cholerae. The stool and water were collected by the microbiological team for sero typing and taken to their lab in Biratnagar for analysis. Stool culture and use of antisera test used in our study led to identification of serotype. Agglutination in 0 group 1 -specific antiserum directed against the lipopolysaccharide component of the cell wall and remove by demonstration of their enterotoxigenicity was done for serotyping.

\section{DISCUSSION}

Table 1. Age wise distribution of cases

\begin{tabular}{lcc}
\hline Age Group (years) & Frequency & Percentage \\
\hline $0-10$ & 61 & 40.6 \\
$11-20$ & 16 & 10.6 \\
$21-30$ & 9 & 6.0 \\
$31-40$ & 13 & 8.6 \\
$41-50$ & 7 & 4.6 \\
$51-60$ & 19 & 12.6 \\
$>60$ & 25 & 16.6 \\
Total & 150 & 100.0 \\
\hline
\end{tabular}

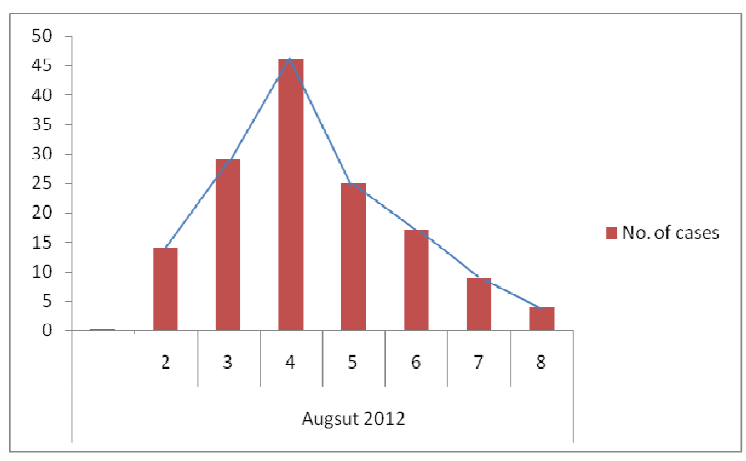

Figure 1. Epidemiological curve

In an underdeveloped country like ours, where adequate sanitary hygiene isn't practiced; cholera is found to be a major public health problem especially among children and elderly. This finding contradictated to the finding by DR Bhatta and Taneja ${ }^{6,7}$ but was consistent to the finding by Palpasa and Bhandari., 5

Cholera outbreak usually occurs in rainy season or the monsoon season. This pattern of disease occurrence was noted in all south Asian countries and countries in tropical region. This was also observed in our study too. ${ }^{3,8}$ In our study, water tank was found to be the source of infection and consumers, mostly children and elderly were affected. Besides that, lack of proper hygiene practices led to amplification of the scenario. The cluster of cases was observed within a short span of duration and the lab finding of the causative agent in both stool and water was consistent. It was also noted that the occurrence of cases declined after chlorination of tank, cleaning of the water source and tank, health education, medication and demonstration of hygiene practices to the mother group by the rapid response team. In a study done in India, outbreak of cholera was attributed to washing feces, drinking and 
bathing. ${ }^{7}$ Vibrio Cholera can survive in water for month to years along with the aquatic plants retaining the ability to infect human where possible. ${ }^{9}$ This was consistent to the observation made about the water tank in our study.

Although we could confirm the outbreak by history and lab investigation but couldn't attribute it completely to the environmental and personal factors due to lack of controls in this study. This was the main limitation of this study. The control couldn't be included due to lack of manpower and fund.

\section{CONCLUSIONS}

Contaminated water tank was found the main source of infection and Vibrio Cholerae 01 El Tor, Ogawa serotype were identified to be the main causative agent behind the outbreak of diarrhea in Shantinagar. Several demonstrations were shown to the villagers and hands on experience about cleaning the tank were provided to the members of water management committee. Cleaning and chlorination of tank was also recommended quarterly and the health information and education was provided to the mothers about the importance of sanitary hygiene and practices.

\section{ACKNOWLEDGEMENTS}

Our sincere acknowledgement to Mr. Chabi Khatiwoda, (Ilam DHO), Dr. Bharati Shakya, Dr. Kumar Gaurav, Dr. Pranil Man Singh Pradhan and Dr. Sabin Shrestha for their cooperation and sincere input by Sandesh Rayamajhi. We are also thankful to B. P. Koirala Institute of Health Sciences for the valuable time.

CONFLICT OF INTEREST: None to declare.

FINANCIAL INTEREST: None to declare.

\section{REFERENCES}

1. Murugaiah C. The burden of Cholera. Crit rev Microbiol 2011;37:337-348.

2. Jason B Harris RCL, Firdausi Qadri, Edward T Ryan, Stephen B C alderhood. Cholera Lancet 2012;379:24662476.

3. Bhandari GP, Ghimire U, Maskey MK. Outbreak Investigation of Diarrheal Diseases in Jajrkot. J Nepal Health Res Counc 2009; 7:66-78.
4. Bhandari GP. Diarrhoeal Disease a neglected epidemics in Nepal. In: Bhandari GP SS, Sharma A, editor. National Workshop on prevention and control of diarrheal diseases 2011; Kathmandu: Nepal Public Health Foundation; 2011.

5. Kansakar PB, Malla S, Ghimire GR. Antimicrobial susceptibilities of enteric bacterial pathogens isolated in Kathamndu, Nepal during 2002 -2004. J infect Dev Countries 2011;5:163168.

6. Raj K SJ, Bhatta DR. Study of enteropathogens and its predisposing factors in gastroenteritis suspected children attending Kanti Children Hospital, Kathamndu, Nepal. J Nepal Assoc Med Lab Sci 2004;6:48-53.

7. Taneja NMB, Khurana S, Sharma M. Antimicrobial resistance in selected bacterial enteropathogens in North India. Ind J Med Res 2004;120:39-43.

8. Michael CF, Sirajul IM. Seasonality of Cholera from 1974 to 2005: A review of global patterns. Int J Health Geographics 2008;7:31.

9. Codec CT. Endemic and epidemic dynamics of cholera: the role of the aquatic reservoir. BMC Infect Dis 2001;1:1.

\section{Citing this article}

Rayamajhi RB, Pokharel G, Sharma G,et al. Outbreak investigation of Cholera in Shantinagar VDC of Ilam District in Eastern Nepal. Int J Infect Microbiol 2013;2(3):87-90. 